\title{
The role of integrated behavioral health in caring for patients with metabolic disorders
}

\author{
Neelkamal Soares, Roger W. Apple, Shibani Kanungo \\ Department of Pediatric and Adolescent Medicine, Western Michigan University Homer Stryker MD School of Medicine, Kalamazoo, MI, USA \\ Contributions: (I) Conception and design: All authors; (II) Administrative support: None; (III) Provision of study materials or patients: None; (IV) \\ Collection and assembly of data: None; (V) Data analysis and interpretation: None; (VI) Manuscript writing: All authors; (VII) Final approval of \\ manuscript: All authors. \\ Correspondence to: Neelkamal Soares, MD. Department of Pediatric and Adolescent Medicine, Western Michigan University Homer Stryker MD \\ School of Medicine, 1000 Oakland Drive, Kalamazoo, MI 49008, USA. Email: neelkamal.soares@med.wmich.edu.
}

\begin{abstract}
This review of integrated behavioral health (IBH) provides a background on IBH models, and the benefits of IBH in pediatric practice with an emphasis on how IBH specialists can collaborate with families and clinicians. An overview of intellectual disability (ID) and psychiatric disorders focused on disorders of inborn errors of metabolism (IEM) highlights issues in assessment and monitoring of these patients with implications for clinical practice and the role of IBH in caring for patients with IEM disorders.
\end{abstract}

Keywords: Integrated behavioral health (IBH); intellectual disability (ID); metabolic disorders

Submitted Oct 19, 2018. Accepted for publication Oct 24, 2018.

doi: $10.21037 /$ atm.2018.10.62

View this article at: http://dx.doi.org/10.21037/atm.2018.10.62

\section{Introduction}

Many children and adolescents with developmental, behavioral or mental health concerns may not receive appropriate evaluation and treatment from their primary care physicians largely due to physicians not having adequate time, comfort level or expertise to adequately address, identify, and treat this type of concern (1-5). It is estimated that approximately 20 percent of children in the United States have a diagnosable mental health condition, yet only about 30 percent of those ever receive treatment $(3,5)$. It is estimated that by the year 2020 , the combination of mental health and substance abuse problems may surpass physical health problems (2). This makes integrating behavioral health services into pediatric care essential in order to address the growing demand as well as allow pediatric practices to continue to run efficiently (6).

\section{Integrated behavioral health (IBH)}

The terms "behavioral health" and "mental health" are often used interchangeably $(7,8)$. While "mental health" is likely more familiar to the general public, "behavioral health" while including behavior problems as presenting concerns, also encompasses the psychological and behavioral factors associated with acute illness and chronic diseases, including factors such as adaptation of the patient, caregiver and family to chronic disease or treatment adherence by the patient.

Recent adaptation of clinics to the patient centered medical home (PCMH) model has resulted in an incorporation of these healthcare components into a primary care setting. Previously, behavioral health services and pediatric practices functioned as distinct and separate entities at separate locations, utilizing a referral system for pediatric patients needing behavioral health services. This model is problematic, because not all physicians are able to accurately identify many behavioral health conditions and approximately $50 \%$ of children and adolescents will not complete an external referral (3). Despite becoming essential components to many pediatric practices; IBH services are often not well defined and can look very 
different across practices, however the most common models for IBH services are coordinated, co-located, or fully integrated services $(2,5,9,10)$.

\section{Coordinated}

Coordinated care models involve facilitating communication across disciplines, such as referring to mental health providers, exchange of medical records, and communicating over the phone. This model is the lowest level of integration, but for some pediatric practices may be preferable to hiring full time behavioral health consultants (BHCs).

\section{Co-located}

Co-located models are becoming more common, where medical and behavioral health providers co-exist in the same location, but otherwise operate as traditionally separate practices with the pediatric practice referring patients to the behavioral health service. Being co-located provides an excellent resource to both the pediatricians and BHCs to collaborate and exchange information when necessary, particularly if sharing the same health record (electronic or otherwise).

\section{Fully integrated/collaborative}

Fully integrated models consider BHCs as part of the medical team working side-by-side with pediatricians during scheduled patient visits. In fully integrated models, the purpose of IBH services is not to replace traditional mental health systems, but rather, to give medical practices an increased ability to effectively respond to behavioral health concerns in efficient, cost effective and timely manner $(9,10)$.

\section{Hybrid approach}

Sometimes there might be a need for a combination of the three primary IBH models (coordinated, co-located, and fully integrated/collaborative) to meet the needs of the patient. In such a situation, a hybrid approach would be most appropriate (e.g., collaborative model providing anxiety intervention and coordinated care with psychiatry). It is critical to remember that the needs of the patients and practice should determine which type of model is appropriate, including a hybrid approach (5). Hybrid models highlight the very diverse set of services provided by IBH and that IBH models should not be viewed as one- size-fits-all.

\section{Role of BHCs}

BHCs who implement IBH services are often trained at the master's level and they can come from various professions including psychology, social work, or counseling (9). Each practice setting varies in use of BHCs; some having a BHC see every scheduled patient, while others have BHCs only see patients with an identified behavioral health concern. BHCs offer brief consultation within the time frame of the medical appointment to address a number of concerns $(9,10)$. BHCs may offer follow-up appointments to address issues that require additional time beyond the medical appointment. These visits are not traditional psychotherapy sessions, typically last a maximum of 30 minutes, and take place in an exam room just like any other appointment scheduled at the practice (9).

\section{Benefits of IBH}

IBH provided in pediatric practice, enables practices to offer behavioral health screening, assessment, and evidence-based treatments. IBH services allow for ease of access to medical and behavioral health services, improved medical and behavioral health outcomes, and improved educational experience for trainees in an academic setting (1,3-6). Additionally, BHCs and pediatricians working in collaboration with children and families has been shown to be effective in improving patient outcomes (1,3-5).

IBH services are considered by some to be an efficient way to deliver care due to many factors including high demand in pediatric practice; quick and easy access to psychologists resulting in earlier diagnosis; management, rapid assessment and intervention; and improved quality and continuity of intervention. IBH services also allow pediatricians more time to focus on other aspects of medical care, results in decreased cost, increase in physical health follow up, increased physician confidence in treating behavioral health concerns, and decreased stigma of behavioral health concerns (6).

\section{Intellectual disability (ID)}

ID occurs in approximately $1 \%$ of the general population characterized by deficits in intellectual and adaptive functioning with severity levels based on adaptive functioning rather than intellectual quotient (IQ) score (11). 
While BHCs generally do not conduct comprehensive psychological assessment, they can help identify patients with ID in subspecialty clinics by administering intellectual assessments as part of their role. The archaic term "mental retardation" was largely replaced since 2007 (12) and is part of the group of intellectual disorders, namely ID, global developmental delay, and unspecified ID (11). A diagnosis of ID is made if onset occurs during the "developmental period" (generally first 8 years of life), and requires deficits in intellectual and adaptive functioning that result in failure to meet developmental and sociocultural standards. Assessment involves more than simply administering an IQ test, but also generally involves a clinical interview, administering adaptive measures, and sorting through possible differential diagnoses. The process often involves review of prior records (medical, educational, psychological) and eliciting family history (including mental health history), psychosocial functioning, exposure to violence, exposure to substances, trauma history, academic functioning, developmental and medical history. It is important that the psychologist conducting the evaluation have training in reliable and valid assessment procedures and significant experience with individuals with ID. This ensures both the quality of the assessment results and the accuracy of severity levels identification. Providers should also pay close attention to the impact of culture, gender, stigma, and socio-economic status on intellectual functioning $(13,14)$.

Psychometric assessment of ID is essential, as there are many confounding variables that can impair observation or conversation with a patient such as comorbid diagnosis of autism spectrum disorder (ASD), in which social impairments make observational diagnosis of ID challenging. Common measures of intelligence are normreferenced intelligence tests, which provide a score said to represent global intelligence. They are generally comprised of tasks or subtests that are administered in a standardized manner. Standards are established using large groups of people (preferably with similar characteristics such as race and socioeconomic status). See Table 1 for commonly used tests. Brief measures of intelligence such as the Wechsler Abbreviated Scale of Intelligence (WASI) and the Kaufman Brief Intelligence Test (K-BIT2) can be used if comprehensive assessments are not possible. For individuals who are non-verbal, specific non-verbal intelligence tests like the Test of Non-verbal Intelligence (TONI-4) and Leiter International Performance Scale, Third Edition (Leiter-3) should be used.
Adaptive functioning measures include individually administered surveys, as well as other sources of relevant information focusing on significant limitations in one or more of the three adaptive skill areas (conceptual, social, and practical). These are also standardized measures normalized on the general population, including people with disabilities and people without disabilities (15). Some examples of adaptive measures include the Vineland Adaptive Behavior Scales, the Diagnostic Adaptive Behavior Scale and the Adaptive Behavior Assessment System (ABAS-III). More recently, adaptive behavior measures are considered equivalent to intellectual results in terms of the understanding and diagnosis of ID and also provide a framework for developing person-centered goals and planning for support (15).

IQ scores and adaptive scores are not as "static" as once assumed, as maturation and experience may impact the results. Additionally, tests for younger children often address different skills than tests for adolescents and standardization of different versions of instruments also affects scores over time. Individual tests differ in what they measure and how, so scores from two different published tests may be vary as can results in the same child when giving a verbal vs. nonverbal test (e.g., in ASD).

\section{ID and inborn errors of metabolism (IEM)}

Disorders of IEM account for about 1-5\% of unspecific ID, with approximately 90 different IEM disorders associated with ID (16). While genetic etiology testing is part of practice guidelines from the American Academy of Pediatrics (17) and American College of Medical Genetics (18), metabolic studies are generally not considered "first line" of investigations in the absence of findings that are highly suggestive such as developmental regression, worsening neurological symptoms, lethargy, seizures or lab findings such as acidosis (18). Focused or sequential metabolic testing (i.e., based on results of basic tests) can increase the diagnostic yield up to $14 \%$ (19). Relatively homogeneous populations can also have a higher yield of individuals positive for a particular IEM diagnosis, e.g., Ashkenazi Jews (20).

Mechanisms by which disorders of IEM might cause neurological effects resulting in ID include alterations in molecular synaptic organization and plasticity mechanisms, influencing expression of genes by toxic consequences in prenatal or early developmental period. Toxic impacts include energy defects, storage of abnormal 
Table 1 Measures used in determining intellectual disability

\begin{tabular}{|c|c|}
\hline Measures & Age range \\
\hline \multicolumn{2}{|l|}{ Intellectual } \\
\hline Wechsler Preschool and Primary Scale of Intelligence-Fourth Edition (WPPSI-IV) & 2 years 6 months to 7 years 7 months \\
\hline Wechsler Intelligence Scale for Children-Fifth Edition (WISC-V) & 6 years to 16 years 11 months \\
\hline Wechsler Adult Intelligence Scale-Fourth Edition (WAIS-IV) & 16 years to 90 years 11 months \\
\hline Woodcock-Johnson Tests of Cognitive Abilities (WJ IV-COG) & 2 to $90+$ years \\
\hline Kaufman Brief Intelligence Test, Second Edition (KBIT-2) & $4-90$ years \\
\hline Wechsler Abbreviated Scale of Intelligence (WASI) & $6-90$ years \\
\hline Test of Nonverbal Intelligence, Fourth Edition (TONI-4) & 6-89 years \\
\hline Interview form, parent/caregiver form & 0-90 years \\
\hline Teacher form & $3-21$ years \\
\hline \multicolumn{2}{|l|}{ Adaptive Behavior Assessment System, Third Edition (ABAS-3) } \\
\hline Parent/primary caregiver form & $0-5$ years \\
\hline Teacher/daycare provider form & $2-5$ years \\
\hline Parent form & $5-21$ years \\
\hline Teacher form & $5-21$ years \\
\hline
\end{tabular}

substances or via aberrant neurotransmission (20). These mechanisms are important as in some cases, progression of cognitive decline can be slowed through treatment of the underlying disorders (21). Generally speaking, disorders of IEM are unlikely to cause isolated ID since they tend to be multi-systemic, and generally are viewed as progressive neurodegenerative processes (20). Exceptions are $\mathrm{X}$-linked creatine transporter deficiency syndromes (SLC6A), organic acid disorders such as 4-hydroxybutyric aciduria, succinic semialdehyde dehydrogenase deficiency, L-2-Hydroxyglutaric aciduria, storage disorders such as Sanfilippo disease type B and A (mucopolysaccharidosis type IIIB/A), or others such as cerebrotendinous xanthomatosis (20).

There are specific caveats in ID assessment in children with disorders of IEM; one of which is the challenge of collecting data, either due to the length of tests and families' willingness to participate, or appropriateness of tests (e.g., some IQ tests have significant language burden in instructions which impacts patients with limited language ability). Another issue is variability of outcomes between disease groups, which limits combining data from disease groups especially if groups only include a few patients (22). Another caveat is that many of the tests have been normed on populations without disorders of IEM and it is unclear how the results apply in this population.

\section{Psychiatric disorders and IEM}

Mental health disorders can occur in patients with IEM disorders due to accumulation of neurotoxic compounds in the central nervous system, through enzymatic defects, or other protein dysfunction; causing disruption of neurodevelopmental processes or impact on neurotransmitter systems (23). These disorders vary in presentation from psychiatric emergencies, including 
delirium (seen in urea cycle defects and porphyria) and acute psychosis (lysosomal storage diseases such as lateonset Tay-Sachs disease and Fabry disease), chronic disorders such as recurrent psychotic attacks and personality changes (homocystinuria, Wilson disease, lysosomal storage disorders) or ASD [metachromatic leukodystrophy, phenylketonuria (PKU), disorders in purine metabolism, organic acidurias, sterol disorders such as SLOS, urea cycle disorders, and mitochondrial disorders] (23).

A caveat in the management of psychiatric disorders cooccurring in patients with IEM is that some of the symptoms may become "treatment-resistant" as the underpinning neurobiology differs from the primary psychiatric illness. Also, since many IEMs are progressive, patients may initially respond to standard treatments, but this response may reduce over time as neurobiological changes occur (24). Since IEM disorders affect neurotransmitters as well, there might be increased risk of side-effects of psychiatric treatments, and some side-effects may worsen the cognitive picture (such as anticholinergics) while some psychotropic medications may actually worse the presentation of an IEM (such as urea cycle disorders) (24).

\section{Role of IBH in IEM}

While BHCs are of value in primary care practices, they can also serve important roles in pediatric subspecialty practices including practices for chronic conditions such as diabetes, cystic fibrosis, hematologic-oncologic conditions, or comprehensive care of individuals with IEMs. In practices without the resources of an established team of social workers, psychologists and others, BHCs can serve additional roles by screening for behavioral/mental health issues, providing strategies for coping, providing guidance/ advocacy for external resources such as educational supports, social networks and parent support groups, and by addressing barriers to adherence to treatment plans.

Children with IEM disorders are known to have cognitive and social-emotional problems which are commonly under-diagnosed or insufficiently treated by primary care physicians, in part due to lack of identification and limited access to behavioral health professionals (25). Hence, there is a need to reliably screen for these concerns, both to identify children in need of specialized interventions but also to track responses to treatment regimens. Parents of children with IEM often need assistance during times of change for their children, including transitions into school, adolescence and social challenges (26). Adherence to diets also poses a challenge, for example in PKU, behavioral strategies can be effective in achieving long-term dietary adherence in PKU (27). All of these health concerns can be addressed by BHCs in partnership with clinicians and families. Additionally, BHCs can help select appropriate cognitive tests and administer adaptive measures to patients with IEM disorders. This information can inform not only clinicians in ongoing care but help in determinations for supports (levels and types) from community agencies and corroborate school information and help in coordination of care.

\section{Conclusions}

IBH can be extremely valuable in both pediatric primary care, and specialty care like clinical services for patients with IEM. Various models of IBH exist (e.g., coordinated, co-located, fully integrated/collaborative, or hybrid) and can be tailored to the unique needs of each practice. IBH provides IEM clinics with the ability to address various aspects of care of children and adolescents with comorbid ID and/or psychiatric disorders by conducting appropriate brief interventions, behavioral health screenings or adaptive/cognitive measures. These support the activities of the medical clinicians and allow for an interprofessional experience both for patient care and education, enhancing the patient and family experience.

\section{Acknowledgements}

None.

\section{Footnote}

Conflicts of Interest: The authors have no conflicts of interest to declare.

\section{References}

1. Schlesinger AB. Behavioral Health Integration in Large Multi-group Pediatric Practice. Curr Psychiatry Rep 2017;19:19.

2. Crowley RA, Kirschner N, Health and Public Policy Committee of the American College of Physicians. The integration of care for mental health, substance abuse, and other behavioral health conditions into primary care: executive summary of an American College of Physicians position paper. Ann Intern Med 2015;163:298-9.

3. Pisani AR, LeRoux P, Siegel DM. Educating Residents 
in Behavioral Health Care and Collaboration: Integrated Clinical Training of Pediatric Residents and Psychology Fellows. Acad Med 2011;86:166-73.

4. Garfunkel LC, Pisani AR, leRoux P, et al. Educating residents in behavioral health care and collaboration: comparison of conventional and integrated training models. Acad Med 2011;86:174-9.

5. Njoroge WFM, Hostutler CA, Schwartz BS, et al. Integrated Behavioral Health in Pediatric Primary Care. Curr Psychiatry Rep 2016;18:106.

6. Hine JF, Grennan AQ, Menousek KM, et al. Physician Satisfaction With Integrated Behavioral Health in Pediatric Primary Care. J Prim Care Community Health 2017;8:89-93.

7. Peek CJ, the National Integration Academy Council. Lexicon for Behavioral Health and Primary Care Integration: Concepts and Definitions Developed by Expert Consensus. AHRQ Publication No.13-IP001-EF. Rockville, MD: 2013. Rockville, MD; 2013. Available online: http:// integrationacademy.ahrq.gov/sites/default/files/Lexicon.pdf

8. Agency for Healthcare Research and Quality. The Academy for Integrating Behavioral Health and Primary Care. 2013 [cited 2018 Aug 18]. Available online: http:// integrationacademy.ahrq.gov/

9. Robinson PJ, Reiter JT. Behavioral consultation and primary care : a guide to integrating services. 2 nd ed. Springer International Publishing, 2016.

10. Hunter CL, Goodie JL, Oordt MS, et al. Integrated behavioral health in primary care : step-by-step guidance for assessment and intervention 2nd ed. Washington, D.C.: American Psychological Association, 2009.

11. American Psychiatric Association. Diagnostic and Statistical Manual of Mental Disorders (DSM-5). Washington, D.C.: American Psychiatric Pub, 2013.

12. Schalock RL, Luckasson RA, Shogren KA. The renaming of mental retardation: Understanding the change to the term intellectual disability. Intellect Dev Disabil 2007;45:116-24.

13. Thomas-Presswood TN, Sasso J, Gin G. Cultural issues in the intellectual assessment of children from diverse cultural backgrounds. J Soc Distress Homeless 1997;6:113-27.

14. Werner S, Corrigan P, Ditchman N, et al. Stigma and intellectual disability: a review of related measures and future directions. Res Dev Disabil 2012;33:748-65.

15. Tassé MJ, Schalock RL, Balboni G, et al. The Construct of Adaptive Behavior: Its Conceptualization, Measurement, and Use in the Field of Intellectual Disability. Am J Intellect Dev Disabil 2012;117:291-303.

16. van Karnebeek CDM, Shevell M, Zschocke J, et al. The metabolic evaluation of the child with an intellectual developmental disorder: Diagnostic algorithm for identification of treatable causes and new digital resource. Mol Genet Metab 2014;111:428-38.

17. Moeschler JB, Shevell M. Comprehensive Evaluation of the Child With Intellectual Disability or Global Developmental Delays. Pediatrics 2014;134:e903-18.

18. Schaefer GB, Mendelsohn NJ. Clinical genetics evaluation in identifying the etiology of autism spectrum disorders: 2013 guideline revisions. Genet Med 2013;15:399-407.

19. Papavasiliou AS, Bazigou H, Paraskevoulakos E, et al. Neurometabolic testing in developmental delay. J Child Neurol 2000;15:620-2.

20. García-Cazorla A, Wolf NI, Serrano M, et al. Mental retardation and inborn errors of metabolism. J Inherit Metab Dis 2009;32:597-608.

21. Peters C, Shapiro EG, Anderson J, et al. Hurler syndrome: II. Outcome of HLA-genotypically identical sibling and HLA-haploidentical related donor bone marrow transplantation in fifty-four children. The Storage Disease Collaborative Study Group. Blood 1998;91:2601-8.

22. Shapiro E, Bernstein J, Adams HR, et al. Neurocognitive clinical outcome assessments for inborn errors of metabolism and other rare conditions. Mol Genet Metab 2016;118:65-9.

23. Simons A, Eyskens F, Glazemakers I, et al. Can psychiatric childhood disorders be due to inborn errors of metabolism? Eur Child Adolesc Psychiatry 2017;26:143-54.

24. Walterfang M, Bonnot O, Mocellin R, et al. The neuropsychiatry of inborn errors of metabolism. J Inherit Metab Dis 2013;36:687-702.

25. Waisbren S, White DA. Screening for cognitive and social-emotional problems in individuals with PKU: Tools for use in the metabolic clinic tr. Mol Genet Metab 2010;99:S96-9.

26. Khangura SD, Tingley K, Chakraborty P, et al. Child and family experiences with inborn errors of metabolism: a qualitative interview study with representatives of patient groups. J Inherit Metab Dis 2016;39:139-47.

27. MacDonald A, Gokmen-Ozel H, van Rijn M, et al. The reality of dietary compliance in the management of phenylketonuria. J Inherit Metab Dis 2010;33:665-70.

Cite this article as: Soares N, Apple RW, Kanungo S. The role of integrated behavioral health in caring for patients with metabolic disorders. Ann Transl Med 2018;6(24):478. doi: 10.21037/atm.2018.10.62 